\title{
TeleProd: An Internet based Teleproductics system
}

\author{
João Paulo N. Firmeza \& Fernando M. S. Ramos \\ Department of Electronics \& Telecommunications - University \\ of Aveiro
}

Campus Universitário de Santiago

3810 Aveiro, PORTUGAL

Tel. $+351-34-370500 \quad$ Fax: $+351-34-370545$

E-Mail Addresses: firmeza@inesca.pt,fmr@inesca.pt

\begin{abstract}
The recent Internet boom and its new powerful development tools are impelling the appearance of new challenging fields of applications to the global network. One new promising field lays on the remote access, control, setup and monitoring of industrial facilities through a computer's network such as the Internet.

TeleProd is a particular implementation built over a proposed reference architecture, that uses the teleproductics concept applied to the Internet network and its associated technologies.

This reference architecture proposes systems where the remote operators connect to virtual reality designed industrial facilities and shop floors using a simple WWW browser. The setup, control, operation and monitoring of the industrial process is accomplished by interacting on a client-server manner with one or several shop floor servers running Microsoft Windows NT. These machines act as bridges between the remote connected clients and the industrial facility itself.

This paper describes, in detail, how current Internet development tools can be used for this application, and results from TeleProd, a research project that is focused on the development of virtual laboratories for the teaching of mechanical engineering.
\end{abstract}

Keywords

Teleproductics, Internet, Client-Server architecture, WWW, Shop Floor Integration, Distance Learning 


\section{INTRODUCTION}

The main issues described on this paper are related to virtual manufacturing and manufacturing integration lying on the teleproductics concept. A particular implementation, TeleProd, developed over a proposed reference architecture (Firmeza, 1997) will also be described in detail. Teleproductics is the interaction among industrial facilities, shop floor devices, computer systems and telecommunications networks. The main idea of application of teleproductics system, is the remote control of self-contained highly automated industrial plants. In despite of the apparent concept's limitation related to more wide application fields, in fact, many applications can be imagined. An example is indeed, the developed system, designed to be used on a distance learning project. The main propose of TeleProd is enabling teaching mechanical engineering by using virtual laboratories (enabling cost reductions due to the overcome of multiplication of similar facilities), using the Internet benefits such as accessibility, easy of use and globalisation (Minoli 1996).

Figure 1 illustrates the concept of teleproductics applied to $\operatorname{IDL}^{[1]}$.

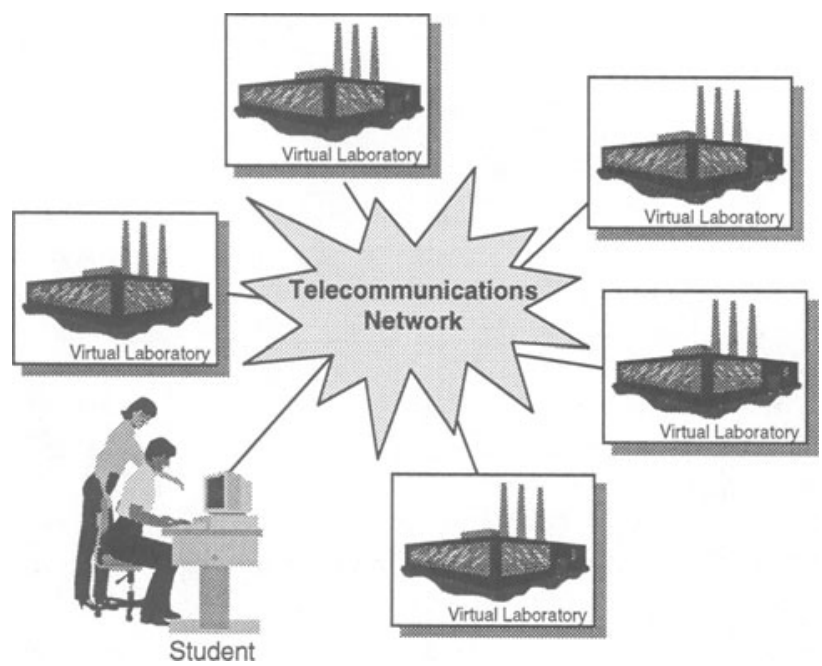

Figure 1: Teleproductics applied to IDL concept.

${ }^{[1]}$ IDL: Interactive Distance Learning 


\section{SYSTEM'S DESIGN AND ARCHITECTURE}

On this item we'll describe the project of an architecture and the implementation approach taken to build a Web based teleproductics system, aimed to demonstrate the capabilities and potentialities of the general concept.

On our particular design, the system will have the following main modules:

- A virtual Control and Operation Platform, designated COP.

- A Manufacturing Integration Platform, designated MIP.

- The Manufacturing Platform, which includes a shop floor containing its specific devices and custom industrial network.

- Feedback circuits, which includes return video data and alarm/event notification.

The next figure presents a simplified block diagram for a reference teleproductics system, and a reference architecture obtained from this specific design:
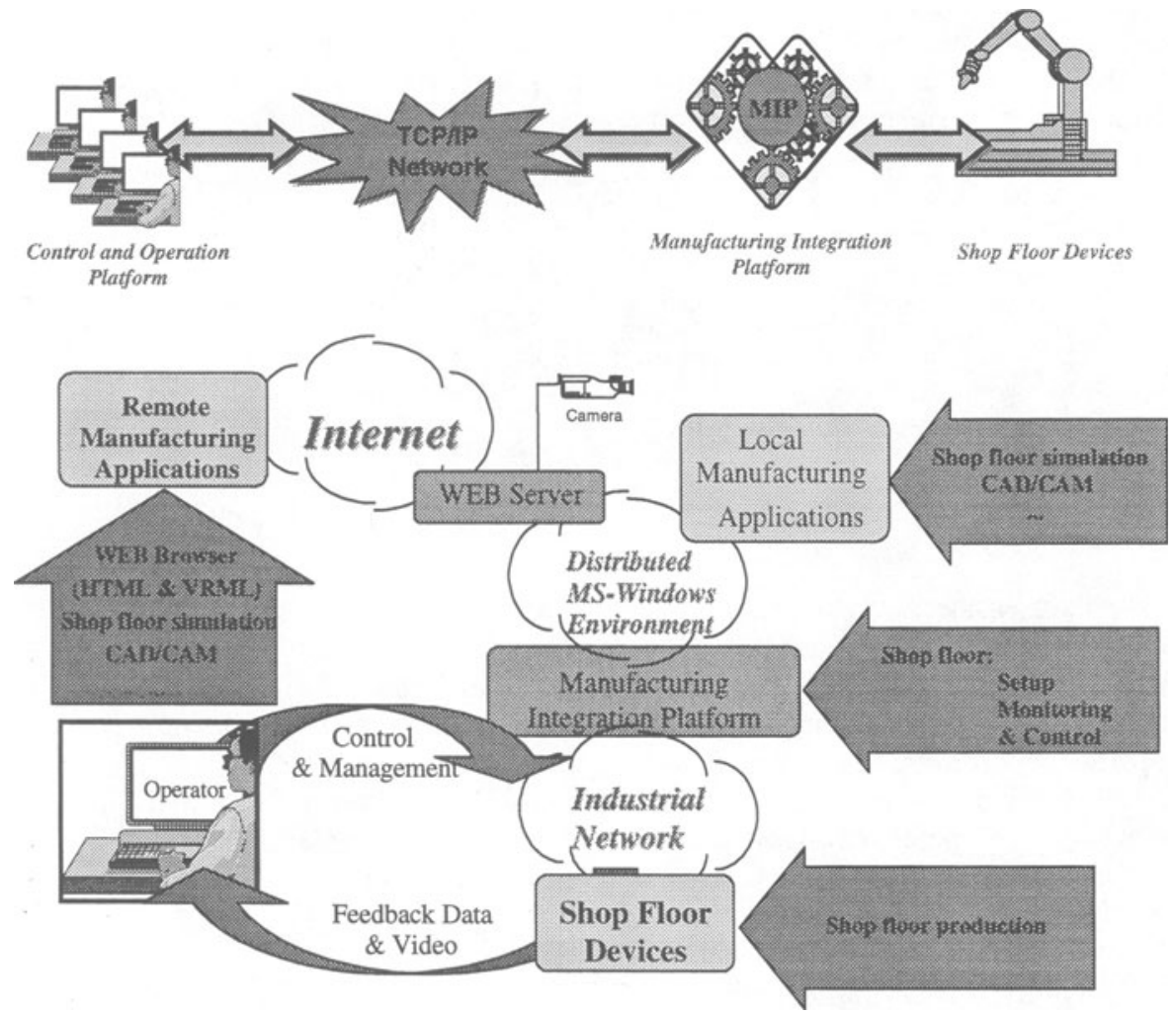

Figure 2: Block diagram and architecture of an Internet based teleproductics system. 


\subsection{The Control and Operation Platform}

The control and operation platform (COP) includes applications for shop floor simulation, CAD/CAM tools and a container for all the tasks related to remote device control, operation and configuration.

In this design proposal, this container is essentially an HTTP $^{[2]}$ client, which is generally an ordinary Web browser, such as Netscape Navigator or Microsoft Internet Explorer. In fact, these are two of the solutions available which fill most of the COP requirements (JAVA support, installable plug-ins, etc). The use of a browser as the container for the user interface improves the architectural independence of this important module, allowing users to access the virtual laboratories using different machines and operating systems. This feature has a key role on the system's portability and accessibility.

When thinking about a Web browser acting as the virtual interface to the system, we may think that everything was built using the HTML ${ }^{[3]}$ language. Although HTML is used, it has a minor paper on the interface functionality, since it was implemented mostly by using virtual reality models constructed using VRML ${ }^{[4]}$, and JAVA applets to do the "real" job of operating the system.

To better understand how the system is remotely accessed, using the Web browser, let's look at the operations from a top-down perspective (Figure 3):
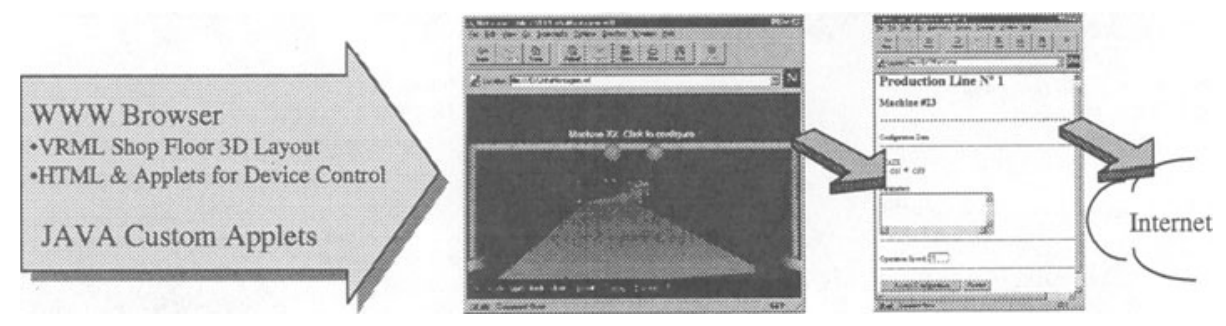

Figure 3: Virtual laboratory and device interaction.

When a user accesses the system by using the Web browser, we gets a VRML 3D model of the virtual laboratory, allowing him to "walk" inside it just by using a pointing device (usually the mouse). When approaching the model of a lab's element or manufacturing device, the user can access a page allowing him to operate or configure the specified device, just by clicking it.

After completion of control data modification, user returns to VRML model, allowing to operate/configure another device. The complete user interaction process is represented on the following diagram (Figure 4):

\footnotetext{
${ }^{[2]}$ HTTP: HyperText Transfer Protocol

${ }^{[3]}$ HTML: HyperText Markup Language

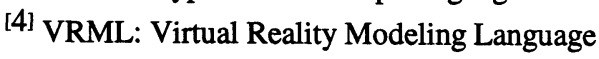




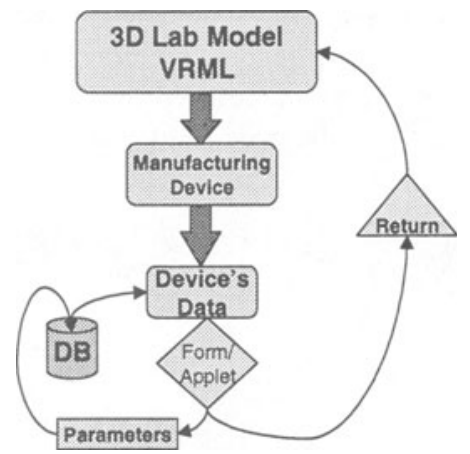

Figure 4: User interaction's top-down diagram.

All the configuration data and shop floor device states are stored on a database placed on a SQL server located at the shop floor facility. This database has an essential role on the entire system, since it acts like a common point of access for both the user modules, and the control and operation services running on the shop floor.

\subsubsection{Control and Operation Modules}

These modules can be implemented either by using HTML forms associated with server CGI applications or ISAs $s^{[5]}$, or by using JAVA applets with remote database connectivity.

On the first approach, all the processing and database access occurs on the lab's side, by remotely launching specific database access applications on HTTP server. These applications tasks are essentially the retrieval of device configuration or state data, and the modification of specified control and operation parameters. The second solution conducts to a more distributed system, since the clients connected to the system do part of the processing work. This JAVA based solution, remotely accesses the database managed by a SQL server placed on the lab, using, for instance, a JBDC ${ }^{[6]}$ connection (Thomas 1996). This solution conducts to a more user-friendly and self-integrated system, containing better features and functionality.

\subsubsection{Device's Native Programs Upload}

Most of the available manufacturing devices need to be programmed to do a specific job. Many allow users to load manufacturing programs, either by using a storage media, or by communicating with the device using a communications network. In the case being studied, we need to upload programs to manufacturing

${ }^{[5]}$ ISA: Internet Server Application. Microsoft's standard for HTTP server applications, built over the Internet Server API (ISAPI).

${ }^{[6]}$ JBDC: JAVA Database connectivity 
devices remotely over the Internet, using the browser. One of the solutions for this problem, and the one being adopted, relays one more time on using JAVA applets. Their task is to upload a user's manufacturing program into a lab's server, and then notify a server's service to deliver the code to the specified device.

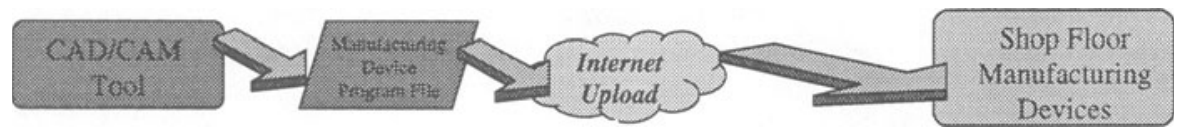

Figure 5: Program file upload process.

\subsubsection{Additional Modules to the Control and Operation Platform}

Integrated, or coexisting with the Web browser, the user needs some additional features. On systems like this one, two additional modules are quite important: an alarms/events reception module, and a video reception module (Wolf 1996).

The alarms/events module is needed to receive alarm messages generated on the lab whenever abnormal or error situations occur on the real manufacturing process. The video reception module is needed to show the real flow of the process, using monitoring cameras installed on strategic placed spots on the shop floor layout.
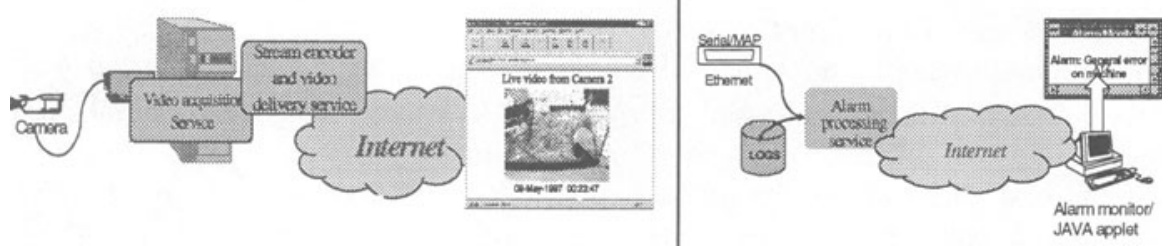

Figure 6: Video and alarms modules.

\subsection{The Manufacturing Integration Platform}

The Manufacturing Integration Platform (MIP) is one of the main system's components. It acts like a bridge between the control and operation clients connected to the manufacturing facility and the facility itself, comprising its shop floor manufacturing devices and overall equipment.

On a logic approach, the MIP must contain a set of services, allowing setup, control and monitoring operations on the shop floor.

A general MIP usually contains several communication interfaces, such as the interface to the virtual manufacturing platforms, remotely connected over a TCP/IP (i.e. Internet), and interfaces to specific manufacturing devices (MAP ${ }^{[7]}$, serial, Ethernet, etc), to support the data flow to all its inner services.

Figure 7 represents the logic architecture of the MIP.

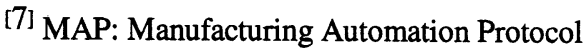




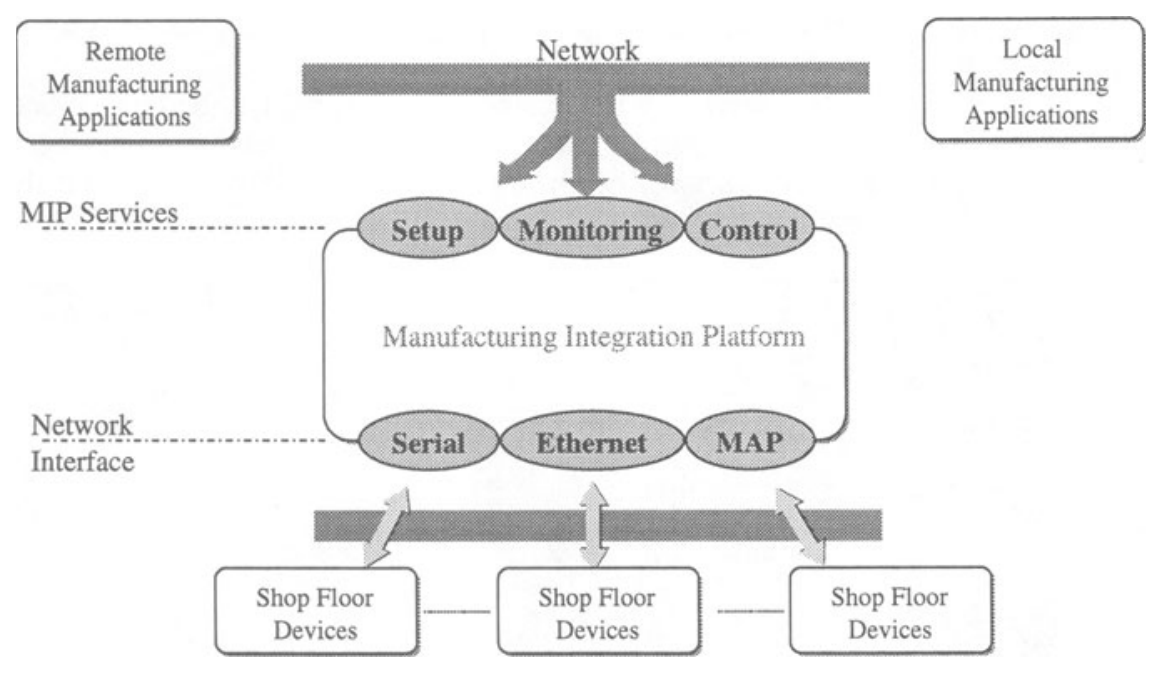

Figure 7: MIP - Logic architecture.

\subsubsection{The Shop Floor: A Windows NT 'Operated' System}

At the shop floor interface with the virtual COPs, the operation's brain is a server (Figure 8), or a server's network, since the main modules can be distributed over several machines. This server, viewed as a logic entity, that we'll call Shop Floor Server, will provide access to several files containing user related data, such as HTML files and VRML model files. Additionally the server is used to store specific applications required to manage the processing of data received from the configuration forms, and other applications such as monitoring and communication tasks. Some of those applications are CGI applications, which are launched by the HTTP service.

Coexisting with the services and associated applications, this machine (or another machine on lab's network) will be the host for a database. The function of this database is the storage and management of the configuration data of the shop floor's components. On the proposed implementation this database is based on a SQL Server, for increased functionality and modularity of the system.

To enable the coexistence of all those services, the operating system to be used on this server must be a multitask operating system, with integrated network support. The proposed solution is based on Windows NT Server, which includes the entire network functionality required embedded. Furthermore its version 4.0 includes also the IIS ${ }^{[8]}$ which includes an HTTP server with support for CGIs, direct access to databases and an API called ISAPI ${ }^{[9]}$, that allows to write applications for the WEB service, with some advantage over CGI applications.

${ }^{[8]}$ IIS: Internet Information Server, from Microsoft Corporation

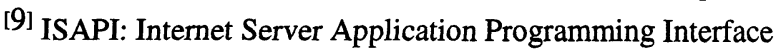


Using the Microsoft SQL Server for Windows $N T$ the connectivity and access to the database problem is also solved.

This platform also hosts a service to process and transmit/receive the control data from/to a central control unit which direct controls specific devices, and also a service to handle the feedback video signals, including its transmission to all the connected management and operation units.

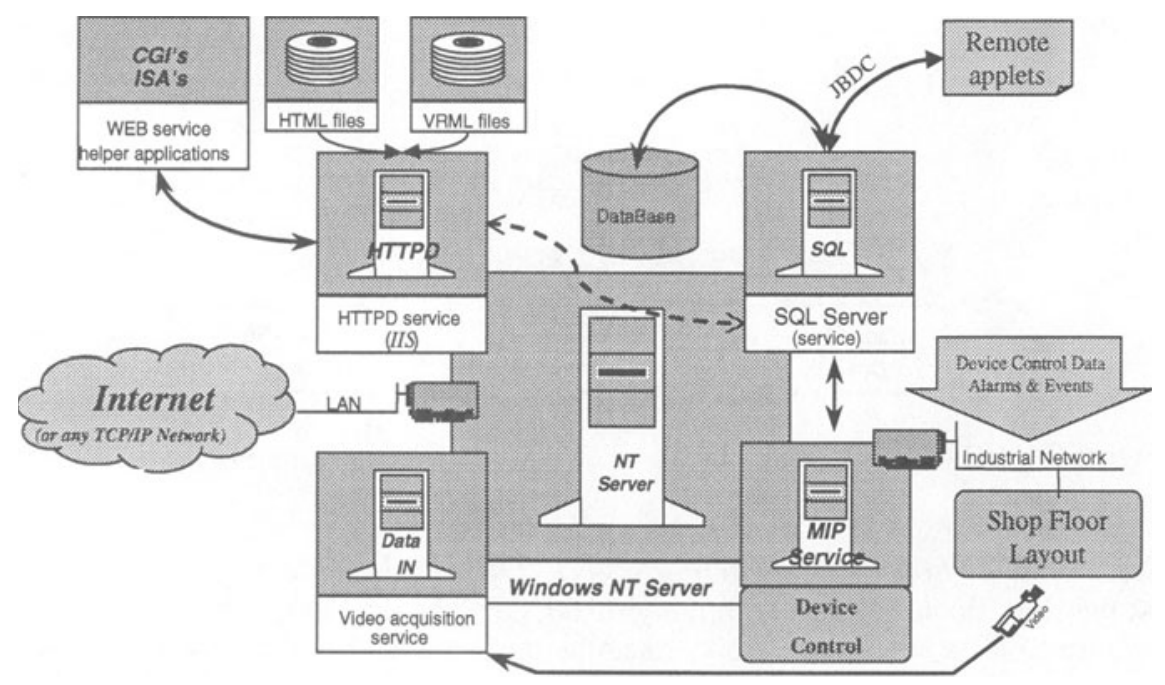

Figure 8: NT Server based MIP.

\subsubsection{Device Control Service \& Interfaces}

Manufacturing devices can present several types of external control interfaces, being the most common serial, MAP/MMS ${ }^{[10]}$ (Messina 1994) or Ethernet interfaces, excluding some proprietary solutions.

A clear issue arises when comparing these and other kinds of interfaces: The addressable ones, and the non-addressable ones. In the first case fits MAP or Ethernet, while serial fits the second case.

The main differences between these two cases are relating to the delivery process to a specified device. While data on addressable devices is kept attached to messages or commands transmitted or received encapsulated on the network protocol used, on non-addressable devices we must have an additional module responsible to data delivery from/to specified devices.

The device control module, named Central Control Unit (CCU), is implemented as a Windows NT service that runs on the server and is always waiting notifications reflecting the occurrence of changes on the system's database. Whenever a user on a control and operation platform performs a database modification, this service receives a message from that client containing a device's identifier. Then, this

${ }^{[10]}$ MMS: Manufacturing Message Specification 
service requests a SQL database query reading the specified device configuration and state. Finally, using that data, the service constructs a frame and delivers a message to the device using the appropriate physical connection, and data encoding.

\subsubsection{Lab's Integration}

The exponential growth of the amount of information switched and stored on a manufacturing process requires a great effort for complete shop floor integration. Some equipment manufacturers, some years ago, started the development of shop floor communication networks based on its proprietary equipment and proprietary defined protocols.

More recently, some efforts are been made in order to promote the compatibility between manufacturing communication systems, and some standards were defined, such as MAP or CNMA. Some generic solutions are also being used, such as Ethernet or centrally managed serial communications.

The proposed architecture puts no restrictions at manufacturing network level, only requiring its existence, and guarantying the complete compatibility with any kind of underlying infrastructure. This way, the architecture fully extends the manufacturing integration concept to outside the facilities, by using a common interaction point with remote operators, accomplished by the shop floor server.

This open architecture allows the distribution of the several processing modules over several machines on the lab's network, including the SQL database, All the remote COPs input/output is accomplished by tunneling data to the shop floor server. (with the eventual exception, depending on the implementation approach, of the video delivery service).

The following figure represents an example of the concept of shop floor integration with a common point of access, using a MAP network to support communications with the shop floor devices.

\section{THE PROTOTYPE TELEPROD}

TeleProd prototype is a demonstration system, created to apply and test the architecture and some of the techniques developed. This prototype system is aimed to be the core of a more complex system used to build a real mechanical engineering distance learning system. TeleProd demo implementation consists of a virtual laboratory built using some very simple resources and devices, such as light bulbs, sensors, actuators, step-motors and cameras, available at the project's work lab. Although these devices may not seem the more appropriate ones to simulate a real mechanical engineering lab's shop floor, they are suitable for testing the most important issues of the system, like remote database interaction, control and monitoring services and global system's integration. This system includes most of the main modules and platforms described on the proposed architecture, although having some limitations related to the work environment present on the lab, like the lack of real programmable manufacturing devices (no CAD/CAM simulation and resulting device program file upload). 


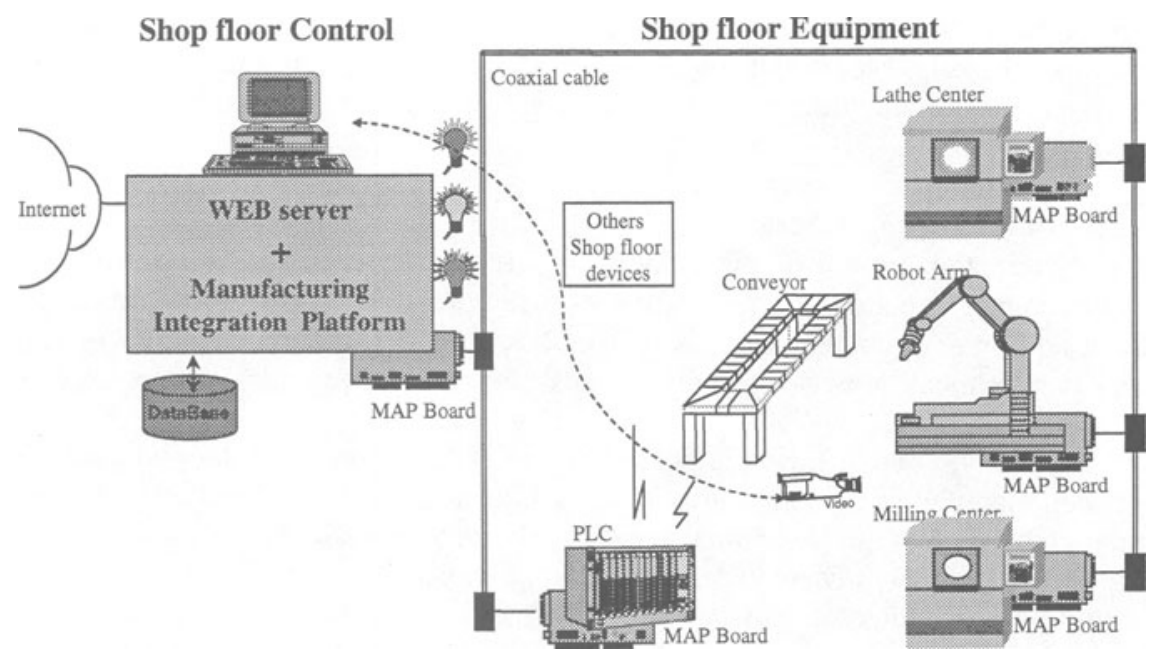

Figure 9: Example of shop floor and system integration.

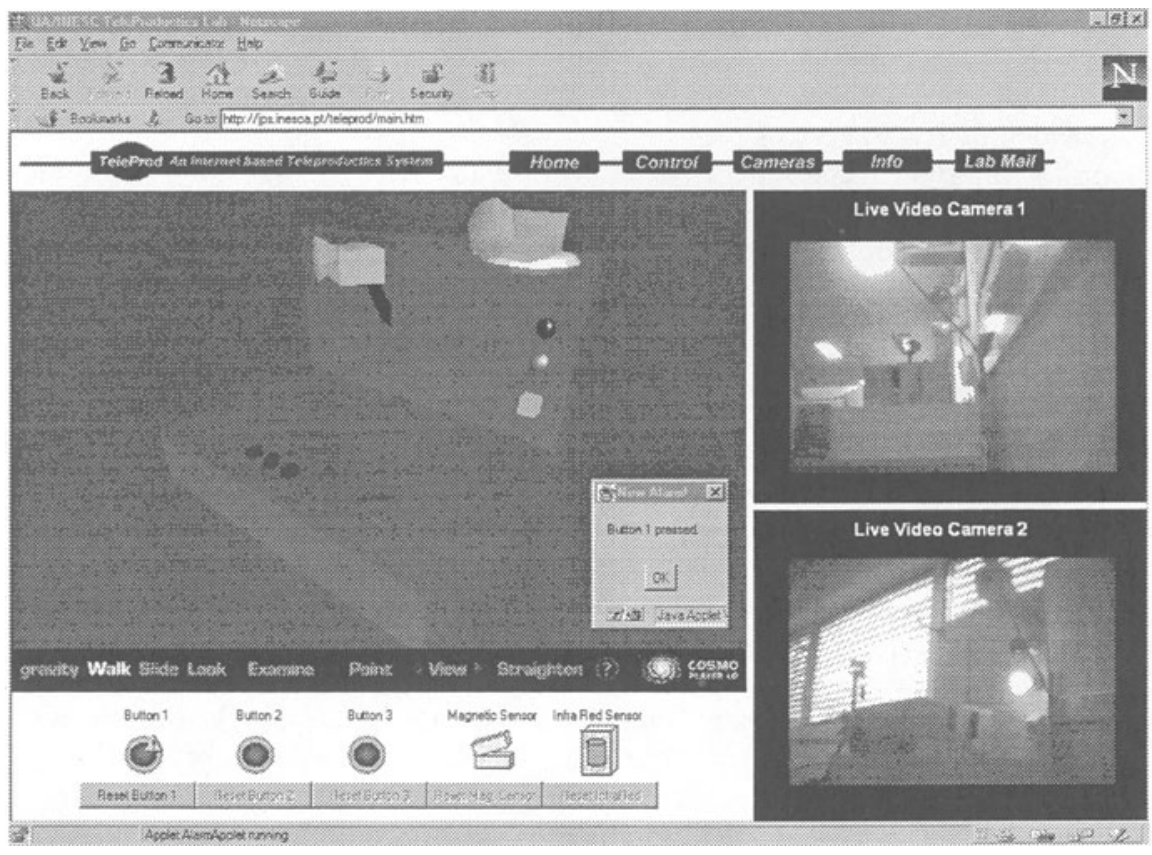

Figure 10: Prototype virtual laboratory. 
On this prototype demo, the control and operation platform was completely built over a Web browser. Users can access the lab by navigating on its 3D model, built using VRML, then they can access each of the individual controllable devices by clicking the mouse. On each case, a specific control page containing remote database access JAVA applets is downloaded from the lab's server. Alarms and events are received using a JAVA reception module that uses TCP/IP sockets. Even the feedback real time video acquired from several sources is presented on the browser client area. Figure 10 shows the general look of the virtual lab graphical interface.

On the middle left frame we can see the lab's device panel 3D model, live video is presented on the right frames and on the bottom frame we can see an alarm reception applet.

Each of the device types is associated with a control applet allowing controlling and changing its parameters. These applets do their job by querying and changing corresponding device's configuration parameters on the database, and then sending a message, using TCP/IP, to a service running on the lab's server.

On the real lab, all control data and messages are managed using a central control unit (CCU) built using a PC with several I/O cards, which is connected to the lab's server using a serial RS-232 connection.

The important thing, is that on the simple device simulated shop floor, the development was entirely made upon the reference architecture for the MIP, as illustrated on the next diagram.

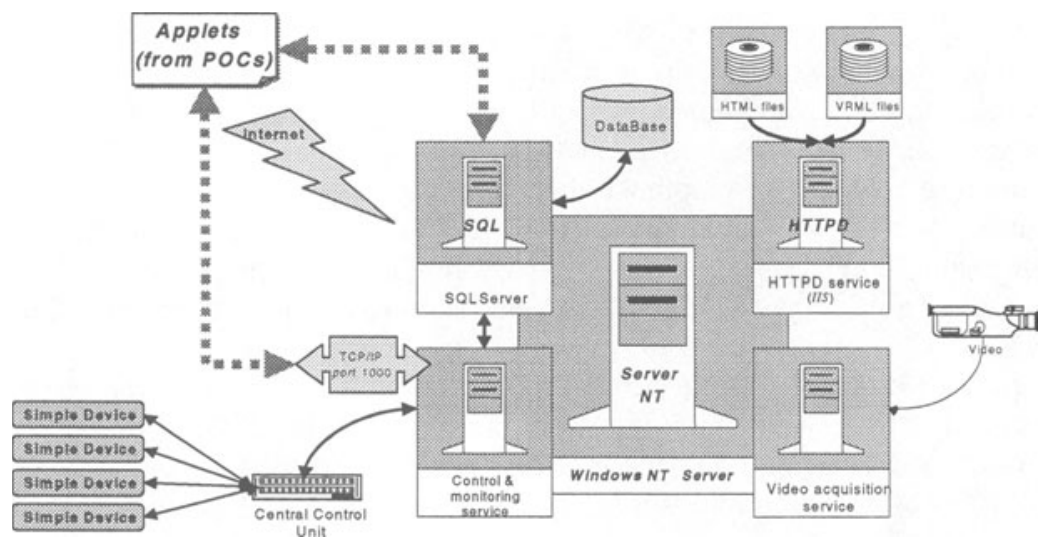

Figure 11: Prototype MIP diagram

\section{CONCLUSION}

Current information and communications technologies built over the Internet, enable the development of new applications that may contribute to better system's integration and substantial cost reductions to industrial corporations: staff costs through the possibility of reusing infrastructures by remotely control existing facilities and investment costs through the capability to increase resource's sharing. 
Teleproductics is a potential example of this, because it will enable remote access to high automated and self contained manufacturing facilities.

This paper discussed how current Internet technologies could be used to implement a Teleproductics system, presenting a running project related to a specific area of application. The system under development is focused to implement virtual laboratories for teaching mechanical engineering.

\section{REFERENCES}

(Firmeza 1997) Firmeza, J. Paulo \& Ramos, Fernando (1997). Designing an Internet based Teleproductics System. Proc. OE/IFIP/IEEE International Conference on Integrated and Sustainable Industrial Production 1997, Chapman \& Hall Press, 325-341.

(Minoli 1996) Minoli, Daniel (1996). Distance Learning Technology and Applications. Artech House 1996.

(Thomas 1996) Thomas, Michael D. (1996). JAVA Programming for the Internet. Ventana: Research Triangle Park.

(Wolf 1996) Wolf, Klaus H \& Froitzheim, Konrad \& Weber, Michael (1996). Interactive Video and Remote Control via the World Wide Web. http://www-vs.informatik.uniulm.de/Papers/IDMS/IDMS.html.

(Messina 1994) Messina, G \& Tricomi, G (1994). Software standardization integration industrial automation systems. Computers in Industry(25) 113-124.

\section{BIOGRAPHY}

Eng. João Paulo N. Firmeza, is a final master student, at the Department of Electronics and Telecommunications, at University of Aveiro. His work for the last two years has been related to telematics, with special focus on teleproductics, covering research, and development of a teleproductics prototype system.

Currently, we is an engineer at CET (Portugal Telecom's Research and Investigation Directorate), where he is researching on broadband access infrastructures, ATM and xDSL technologies and broadband network applications.

Prof. Fernando Ramos, holds a PhD on Telecommunications from the University of Aveiro, Portugal, where he is currently Professor and head of the Department of Electronics and Telecommunications. He is also leading an R\&D group at INESC in the area of telecommunications systems and service. His current research interests include open distance learning and telesurveillance systems based on ISDN, GSM and VSAT. 